\title{
'||-||||||||||||||||||||||||||||||||||||||||||||||||||||||||||||||||||.
}

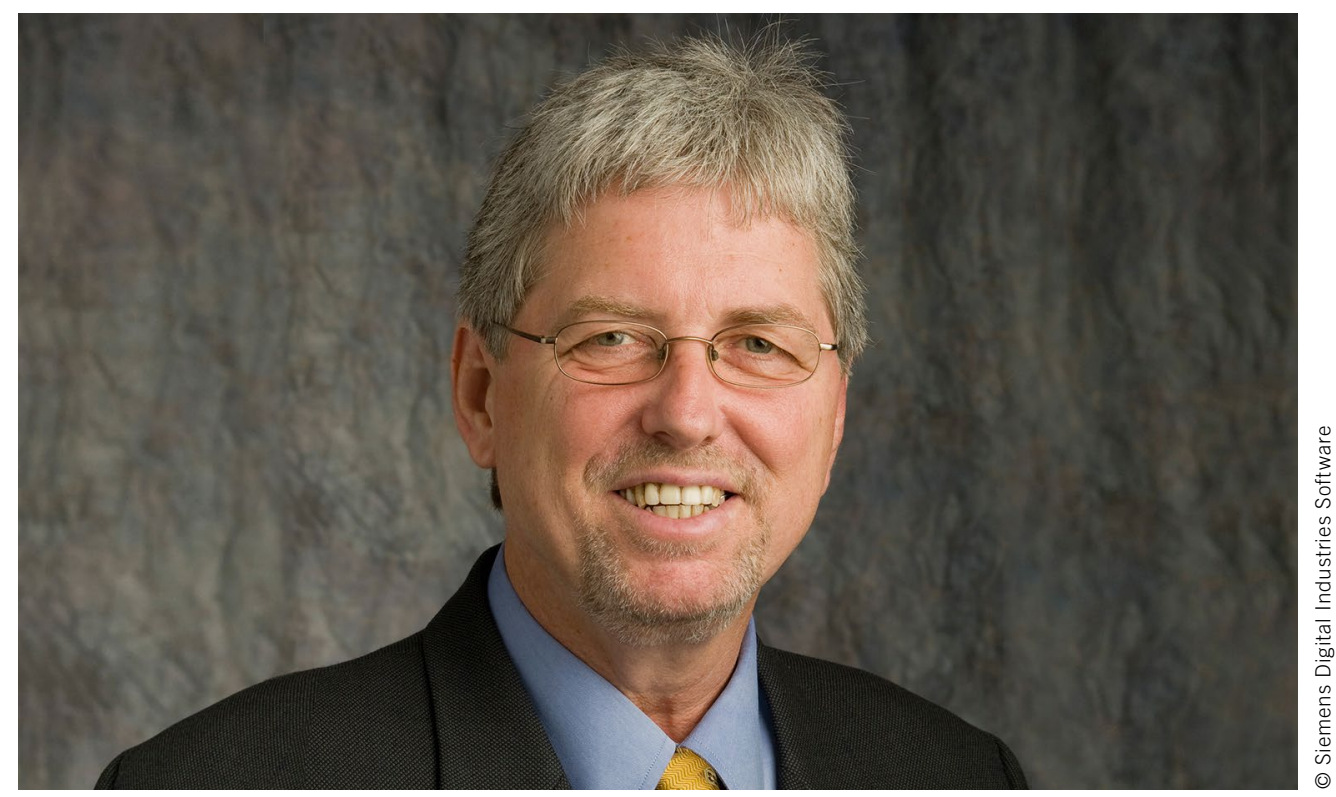

Martin 0'Brien

Senior Vice President Integrated Electrical Systems bei Siemens

Digital Industries Software

\section{Grenzen bei der Entwicklung von E/E-Systemen überbrücken}

Umfassende Elektrifizierung und wachsende Produktintelligenz führen zu einer deutlichen Zunahme der Relevanz und Komplexität von Elektrik-/Elektronik-Systemen (E/E) in einer großen Bandbreite von Produkten. In der Tat hat der schnelle Zuwachs an Komplexität die Vorhersagen bei Weitem übertroffen. Noch vor wenigen Jahren deuteten Schätzungen darauf hin, dass ein durchschnittliches Fahrzeug im Jahr 2020 etwa 30 Millionen Zeilen Softwarecode (SLOC) und 10.000 Netzwerksignale enthalten würde. Gespräche mit unseren Kunden haben jedoch gezeigt, dass die wirklichen Zahlen eher bei 150 Millionen SLOC und 20.000 Netzwerksignalen liegen.

Bei der Produktentwicklung muss jedes untergeordnete System eine vielfältige Kombination von unterschiedlichen Entwicklungsdisziplinen integrieren, um die geforderte Produktfunktionalität zu ermöglichen. Durch diese disziplinübergreifende Komplexität steigt die Herausforderung, eine Rückverfolgbarkeit innerhalb des gesamten Nutzdauerzyklus der Produktentwicklung zu gewährleisten. Ingenieure müssen jetzt Tausende von Komponenten und Funktionen disziplinübergreifend unter Kontrolle behalten, um die Entwicklung, Simulation und Validierung von elektrischen Anlagen, Steuergeräten (ECUs), Softwareanwendungen und weiterem mehr zu koordinieren.

Der traditionelle, isolierte Ansatz bei der Fahrzeugentwicklung - man spricht von „Silos“ - führt häufig zu einer inkonsistenten Umsetzung der Anforderungen beim Konstruieren untergeordneter Systeme, mangelnder Kommunikation zwischen den einzelnen Disziplinen und fehlenden Verweisen bei der Rückverfolgbarkeit von Konstruktionsdaten. Diese Fehlinterpretationen werden häufig erst dann deutlich, wenn verschiedene Systeme miteinander integriert werden, was zu höheren Kosten, Terminüberschreitungen und einem gesteigerten Programmrisiko führt.

Heute und in Zukunft besteht die Notwendigkeit, disziplinübergreifende Integrationen zu unterstützen und die Grenzen zwischen den getrennten Hierarchien (Silos) zu überbrücken, um eine dauerhafte Datenkontinuität während des gesamten Konstruktionsprozesses, der Herstellung und der Instandhaltung von E/E-Systemen zu gewährleisten. Mit diesem Ansatz können Unternehmen die Konstruktion von Elektrik, Elektronik, Netzwerken und eingebetteter Software aus der E/E-Systemarchitektur heraus vorantreiben. Die nachgelagerte Unterstützung für die Bereiche Kabelbaumfertigung und Fahrzeugservice vervollständigt die umfassende E/E-Systementwicklungsumgebung durch das Bereitstellen von Betriebsdaten, die den Entwicklungskreislauf schließen, indem sie in zukünftige Entwurfsiterationen einfließen.

Zukunftsorientierte Unternehmen suchen Partner, die sie dabei unterstützen können, Silos aufzubrechen und die dafür erforderlichen Fähigkeiten in ihre Produktentwicklungsprozesse zu integrieren. Diejenigen, die mit diesen Veränderungen effektiv umgehen können, schaffen sich die besten Voraussetzungen, um in den kommenden Jahren erhebliches Wachstum zu erzielen. 